\title{
Phase II study of concurrent chemoradiotherapy with carboplatin and vinorelbine for locally advanced non-small-cell lung cancer
}

\author{
KOKO ISHIDA ${ }^{1}$, TAKASHI HIROSE ${ }^{2}$, JUNICHI YOKOUCHI ${ }^{3}$, YASUNARI OKI ${ }^{1}$, SOJIRO KUSUMOTO ${ }^{1}$, \\ TOMOHIDE SUGIYAMA ${ }^{1}$, HIROO ISHIDA ${ }^{1}$, TAKAO SHIRAI ${ }^{1}$, MASANAO NAKASHIMA ${ }^{1}$, \\ TOSHIMITSU YAMAOKA ${ }^{4}$, TSUKASA OHNISHI ${ }^{1}$, TOHRU OHMORI ${ }^{4}$ and YOSHIKAZU KAGAMI $^{3}$
}

\begin{abstract}
${ }^{1}$ Division of Respiratory Medicine and Allergology, Department of Internal Medicine, Showa University School of Medicine, Shinagawa, Tokyo 142-8666; ${ }^{2}$ Department of Respirology, National Hospital Organization, Tokyo Hospital, Tokyo 204-8585;

${ }^{3}$ Department of Radiology; ${ }^{4}$ Institute of Molecular Oncology, Showa University School of Medicine, Shinagawa, Tokyo 142-8666, Japan
\end{abstract}

Received October 10, 2012; Accepted January 1, 2014

DOI: $10.3892 / \mathrm{mco} .2014 .252$

\begin{abstract}
Patients with non-small cell lung cancer (NSCLC) have locally advanced disease with poor prognosis. Although concurrent chemoradiotherapy is the standard treatment, more effective regimens are required. The aim of this study was to assess the safety and efficacy of concurrent chemoradiotherapy with a divided schedule of carboplatin and vinorelbine in patients with locally advanced NSCLC. Patients with unresectable, stage IIIA or IIIB NSCLC were eligible for enrollment if they exhibited a performance status of $0-2$ and were $\leq 75$ years of age. Patients were treated with carboplatin at an area under the plasma concentration vs. time curve of $2.5 \mathrm{mg} / \mathrm{ml} / \mathrm{min}$ and vinorelbine at $20 \mathrm{mg} / \mathrm{m}^{2}$ on days 1 and 8 every 3 weeks. Thoracic radiotherapy at a total dose of $60 \mathrm{~Gy}$ was concurrently administered (2 Gy per fraction). Twenty-eight patients ( 23 men and 5 women; median age, 67 years; range $47-75$ years) were enrolled in the present study. The overall response rate was $85.7 \%$ [95\% confidence interval (CI), 67.3-96.0\%] and the disease control rate was $96.4 \%$ (95\% CI, 81.7-99.9\%). The median survival time (MST) was 23 months and the median progression-free survival (PFS) time was 8 months. Grade 3-4 toxicities included neutropenia, thrombocytopenia, anemia and infection in 100, 14, 46 and $36 \%$ of patients, respectively. One patient (4\%) developed grade 3 radiation esophagitis that resolved completely without residual dilation. Grade 3 radiation pneumonitis occurred in 2 patients (7\%); however, the symptoms and radiographic
\end{abstract}

Correspondence to: Dr Takashi Hirose, Department of Respirology, National Hospital Organization, Tokyo Hospital, 3-1-1 Takeoka Kiyose, Tokyo 204-8585, Japan

E-mail: thirose-shw@umin.ac.jp

Key words: carboplatin, chemoradiotherapy, non-small-cell lung cancer, vinorelbine abnormalities subsided with corticosteroid therapy. In conclusion, concurrent chemoradiotherapy with a divided schedule of carboplatin and vinorelbine is well-tolerated and effective in patients with locally advanced NSCLC.

\section{Introduction}

Approximately one-third of patients with non-small-cell lung cancer (NSCLC) have locally advanced disease at the time of diagnosis. The prognosis of locally advanced NSCLC is poor and the 5-year survival rates of patients with clinical stage IIIA and IIIB disease are 18 and 8\%, respectively (1). A meta-analysis of randomized trials demonstrated that platinum-based chemoradiotherapy confers a survival advantage over radiotherapy alone in patients with locally advanced NSCLC (2). Another meta-analysis of randomized trials demonstrated that concurrent chemoradiotherapy improves survival compared to sequential chemoradiotherapy in patients with locally advanced NSCLC (3). Therefore, concurrent chemoradiotherapy has been a standard treatment for patients with locally advanced NSCLC. However, a standard chemoradiotherapy regimen, including radiation dose and schedule as well as selection and dosage of chemotherapeutic agents, has not been determined thus far. Therefore, more investigations are required to develop more effective and less toxic chemoradiotherapeutic regimens for locally advanced NSCLC.

The combination chemotherapy of cisplatin and vinorelbine with concurrent thoracic radiotherapy has been demonstrated to be an effective and tolerable regimen (4). However, the major limitation of this regimen is cisplatin toxicity and the inconvenience of extensive hydration requirements. Carboplatin causes less renal, neurological and gastrointestinal toxicity and is more convenient to administer compared to cisplatin (5). In patients with locally advanced NSCLC, overall survival rates do not differ significantly between carboplatin- and cisplatin-based chemoradiotherapy (6), although previous meta-analyses demonstrated that combination chemotherapy with cisplatin plus a new agent provides a survival advantage 
over carboplatin plus a new agent in patients with metastatic advanced NSCLC $(7,8)$. Therefore, carboplatin-based chemoradiotherapy may be a viable option in patients with locally advanced NSCLC.

Preclinical studies demonstrated that both carboplatin and vinorelbine act as radiation enhancers. Carboplatin enhances the cytotoxic effects of radiation against tumor cells in vitro as well as in vivo (9) and vinorelbine enhances the antitumor effects of radiation in vitro in a cell cycle-dependent manner, with optimal effects when the cells are in the G2/M phase (10). Moreover, previous studies reported that the combination chemotherapy of carboplatin and vinorelbine without thoracic radiotherapy achieves promising outcomes in patients with advanced NSCLC $(11,12)$. However, to date, few trials have evaluated the effectiveness and safety of the combination chemotherapy of carboplatin and vinorelbine with concurrent thoracic radiotherapy in patients with locally advanced $\operatorname{NSCLC}(13,14)$.

In a previous phase I study, Hoffman et al (13) recommended that carboplatin, with a target area under the plasma concentration versus time curve of (AUC) of $3 \mathrm{mg} / \mathrm{ml} / \mathrm{min}$ using the Calvert's formula and vinorelbine, at a dose of $15 \mathrm{mg} / \mathrm{m}^{2}$, be administered on days 1 and 8 every 3 weeks with concurrent thoracic radiotherapy in patients with locally advanced NSCLC. However, this recommended dose of vinorelbine was significantly lower compared to doses in trials without concurrent thoracic radiotherapy in patients with metastatic advanced NSCLC. Furthermore, findings of the phase I/II study by Masters et al (15) suggested that a combination of carboplatin at a target AUC of $2.5 \mathrm{mg} / \mathrm{ml} / \mathrm{min}$ and vinorelbine at a dose of $25 \mathrm{mg} / \mathrm{m}^{2}$ be administered on days 1 and 8 every 3 weeks without concurrent thoracic radiotherapy in patients with metastatic advanced NSCLC. The vinorelbine dose was reduced from 25 to $20 \mathrm{mg} / \mathrm{m}^{2}$ due to concurrent thoracic radiotherapy being added to the chemotherapy. The aim of this phase II study was to assess the antitumor activity and safety of a divided schedule of carboplatin and vinorelbine combined with concurrent thoracic radiotherapy in patients with locally advanced NSCLC.

\section{Patients and methods}

Eligibility criteria. Patients with histologically or cytologically proven unresectable stage IIIA or IIIB NSCLC who had not previously received chemotherapy or radiotherapy were eligible for this study. Other eligibility criteria included: i) age 20-75 years; ii) Eastern Cooperative Oncology Group performance status of $0-2$; iii) a tumor within an estimated irradiation field no larger than half the hemithorax; iv) a measurable lesion; v) life expectancy of $\geq 3$ months; and vi) adequate bone marrow function (white blood cell count of $\geq 4000 / \mu 1$, neutrophil count of $\geq 2000 / \mu 1$, platelet count of $\geq 100,000 / \mu 1$ and hemoglobin level of $\geq 9.0 \mathrm{~g} / \mathrm{dl}$ ), adequate renal (serum creatinine levels $<1.5 \mathrm{mg} / \mathrm{dl}$ and creatinine clearance rate of $\geq 50 \mathrm{ml} / \mathrm{min}$ ) and hepatic function (total serum bilirubin level within the upper limit of the normal range, levels of aspartate and alanine aminotransferase stwice the upper limits of the normal ranges) and arterial oxygen pressure of $\geq 60 \mathrm{mmHg}$. Patients were excluded in case of malignant pleural or pericardial effusion, active infections, severe heart disease, interstitial pneumonia, or an active second malignancy. The study protocol was approved by the Institutional Review Board of Showa University School of Medicine and the patients provided written informed consent.

Treatment schedule. The treatment regimen consisted of carboplatin and vinorelbine with concurrent thoracic radiotherapy. Both carboplatin and vinorelbine were administered on days 1 and 8 . These agents were administered every 3 weeks for a maximum of 4 courses. Vinorelbine at a dose of $20 \mathrm{mg} /$ $\mathrm{m}^{2}$ was diluted in $20 \mathrm{ml}$ of normal saline and administered as an intravenous infusion over $6 \mathrm{~min}$. Carboplatin with a target AUC of $2.5 \mathrm{mg} / \mathrm{ml} / \mathrm{min}$ was diluted in $500 \mathrm{ml}$ of normal saline and administered over $60 \mathrm{~min}$. The carboplatin dose was calculated using the Calvert's formula.

Chemotherapy was discontinued in case of $\geq$ grade 3 non-hematological toxicity, except for nausea/vomiting, anorexia, constipation, diarrhea, esophagitis, alopecia and fatigue; serum creatinine levels $>2.0 \mathrm{mg} / \mathrm{dl}$; a treatment outcome of progressive disease at any time; or an interval of $\geq 2$ weeks after the scheduled initiation of the next course, until the criteria mentioned below were satisfied. Carboplatin and vinorelbine were not administered on day 8 of treatment if the neutrophil count was $<1,000 / \mu 1$ or if the platelet count was $<75,000 / \mu 1$. Full doses of carboplatin and vinorelbine were then administered on day 15 of the treatment. The next course of treatment was initiated after the neutrophil count had increased to $1,500 / \mu 1$; the platelet count had increased to $100,000 / \mu 1$; the creatinine level had decreased to $\leq 1.5 \mathrm{mg} / \mathrm{dl}$; and the non-hematological toxicity, except for anorexia, constipation, alopecia and fatigue, had decreased to $\leq$ grade 2 . The doses of carboplatin and vinorelbine were reduced by a target AUC of $0.5 \mathrm{mg} / \mathrm{ml} / \mathrm{min}$ and $5 \mathrm{mg} / \mathrm{m}^{2}$, respectively, if the patient had grade 4 thrombocytopenia. The dose of vinorelbine was reduced by $5 \mathrm{mg} / \mathrm{m}^{2}$ if the patient had grade 4 neutropenia lasting $\geq 3$ days or grade 3 or 4 neutropenia associated with a temperature of $>38^{\circ} \mathrm{C}$. Prophylactic antiemetic treatment with 5-hydroxytryptamine receptor type 3 antagonists and dexamethasone was routinely administered prior to carboplatin in the patients. If the neutropenia had decreased to grade 4 during chemotherapy, granulocyte colony-stimulating factor (G-CSF) was administered until the neutrophil counts recovered.

Thoracic radiotherapy. Thoracic radiotherapy consisted of standard chest irradiation in single daily fractions of 2 Gy for 6 weeks, up to a total dose of $\sim 60 \mathrm{~Gy}$. The planned initial radiation field was not to exceed $50 \%$ of one lung. The initial dose (up to $40 \mathrm{~Gy}$ ) was administered to the original volume that had been determined with the size and locations of the primary tumor and the draining lymphatic vessels and included a $2 \mathrm{~cm}$ margin around the pretreatment primary tumor and the ipsilateral hilum. The entire width of the mediastinum was included, with a $2 \mathrm{~cm}$ margin around the radiographically visible area of involvement. The inferior margin extended $3 \mathrm{~cm}$ below the carina or $2 \mathrm{~cm}$ below the radiographically visible tumor mass. Subsequently, an additional 20 Gy dose was administered to the boost volume, including the entire primary tumor and clinically involved regional hilar and mediastinal lymph nodes, as determined by computed tomography (CT). The original volume was treated with an anterior-posterior parallel-opposed 
pair of portals and the boost volume was treated with the same pair or with a pair of oblique fields, if the cumulative radiation dose to the spinal cord exceeded $40 \mathrm{~Gy}$. The percentage of lung volume receiving $>20$ Gy (i.e., V20) of radiotherapy was not mandatory in the planning of thoracic radiotherapy.

Thoracic radiotherapy was discontinued for grade 3-4 radiation pneumonitis. Thoracic radiotherapy was suspended for grade 3-4 esophagitis, temperature of $>38^{\circ} \mathrm{C}$ or active infection, during administration of G-CSF, or for a platelet concentration of $<20,000 / \mu 1$ and was resumed when these toxicities had decreased to $\leq$ grade 2 .

Evaluation. Pretreatment evaluation included a baseline history and physical examination, complete blood count with differential, routine chemistry profiles, chest radiography, CT of the chest and abdomen, magnetic resonance or CT imaging of the brain and radionucleotide bone scan. Mediastinoscopy and positron-emission tomography (PET) scans were not considered mandatory for this trial.

Tumor response was classified according to the Response Evaluation Criteria in Solid Tumors version 1.0. Acute toxicities were assessed and graded according to the National Cancer Institute Common Toxicity Criteria version 3.0 and late toxicity associated with thoracic radiotherapy, occurring $>90$ days after the initiation of radiotherapy, was graded according to Radiation Therapy Oncology Group late-toxicity criteria. The patients who received at least 1 cycle of chemotherapy were assessable for response, toxicity and survival.

Statistical analysis. Progression-free survival (PFS) was measured from the initiation of this treatment to the identifiable time of the first progression or death from any cause. Survival time was measured from the initiation of the present treatment until death or last follow-up. The Kaplan-Meier method was used to calculate survival curves. Survival differences between subgroups were compared by means of the log-rank test. $\mathrm{P}<0.05$ was considered to indicate a statistically significant difference.

The present trial was designed as a phase II study, with response rate as the main endpoint. According to Simon's minimax design, our study, with a sample size of 28 , had $90 \%$ power to accept the hypothesis that the true response rate was $>75 \%$ and had a $5 \%$ significance to reject the hypothesis that the true response rate was $<50 \%$.

\section{Results}

Patient characteristics. Between March, 2006 and February, 2010, 28 patients were enrolled (Table I). Thirteen patients had stage IIIA and 15 had stage IIIB disease. Mutations in the epidermal growth factor receptor (EGFR) gene were evaluated in 12 patients. Of these, 3 patients with adenocarcinoma exhibited activating mutations in the EGFR gene. Response, survival and toxicity were assessable in the patients. A total of 102 courses of chemotherapy were administered. The median number of courses given per patient was 4 (range, 1-4).

Treatment response and survival. Out of the 28 patients, $3(10.7 \%)$ achieved a complete response, $21(75.0 \%)$ achieved a
Table I. Patient characteristics.

\begin{tabular}{lc} 
Characteristics & Values \\
\hline Total number of patients & 28 \\
Gender (male/female) & $23 / 5$ \\
Age, years (range) & $67(47-75)$ \\
Performance status (0/1/2) & $5 / 20 / 3$ \\
Stage (IIIA/IIIB) & $13 / 15$ \\
Pathology & \\
Adenocarcinoma & 14 \\
Squamous cell carcinoma & 11 \\
Other & 3
\end{tabular}

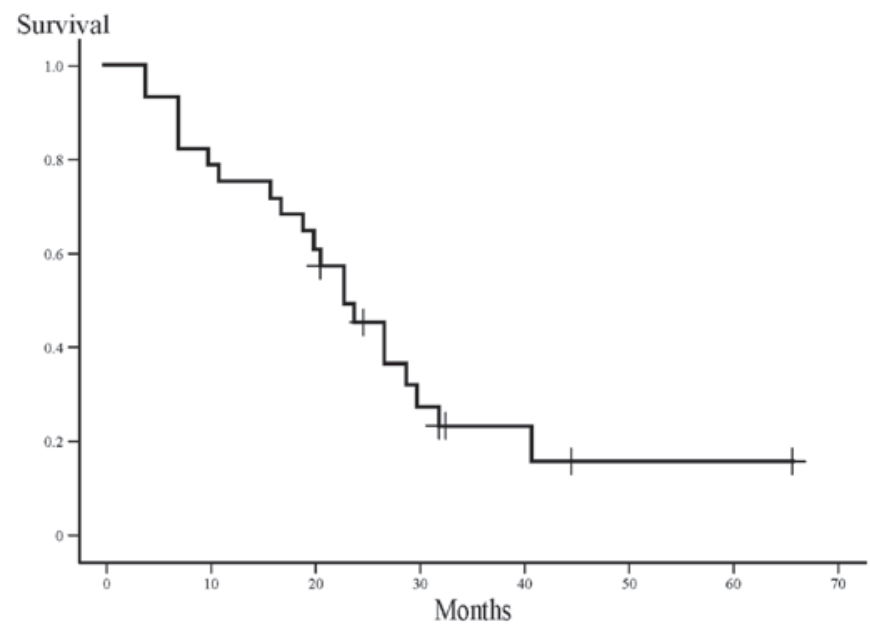

Figure 1. Overall survival estimated with the Kaplan-Meier method. The median survival time (MST) was 23 months (range, 4-66 months).

partial response, $3(10.7 \%)$ had stable disease and $1(3.6 \%)$ had progressive disease, reaching an overall response rate of $85.7 \%$ [95\% confidence interval (CI), 67.3-96.0\%] and a disease control rate of $96.4 \%$ (95\% CI, 81.7-99.9\%).

Survival was analyzed when the median follow-up time of the patients was 22 months. At the time of the analysis, 6 patients $(21 \%)$ were alive and no patients had been lost to follow-up. One patient succumbed to bacterial sepsis during second-line chemotherapy for disease recurrence. The median survival time (MST) was 23 months (range, 4-66 months) and the 2-year survival rate was $43 \%$ (Fig. 1). The median PFS time was 8 months (range, 2-36 months; Fig. 2).

Recurrence pattern and subsequent therapy. At the time of the analysis, $24(85.7 \%)$ out of the 28 patients exhibited disease recurrence. Of these, $8(33.3 \%)$ had local recurrence, $11(45.8 \%)$ had distant recurrence and $5(20.8 \%)$ had local as well as distant recurrence. The most common site of distant metastasis was the brain, followed by intrathoracic sites.

Of these 24 patients, $18(75 \%)$ received second-line chemotherapy; 1 patient (4\%) had a complete response to chemoradiotherapy followed by recurrence at the primary site and surgery; and the remaining 5 patients $(21 \%)$ received only 
Table II. Treatment toxicity.

\begin{tabular}{|c|c|c|c|c|c|}
\hline \multirow[b]{2}{*}{ Toxicity } & \multicolumn{5}{|c|}{ National Cancer Institute common terminology criteria grade } \\
\hline & 1 & 2 & 3 & 4 & $3 / 4(\%)$ \\
\hline Neutropenia & 0 & 0 & 7 & 21 & 100 \\
\hline Thrombocytopenia & 9 & 6 & 4 & 0 & 14 \\
\hline Anemia & 5 & 10 & 10 & 3 & 46 \\
\hline Nausea & 6 & 2 & 0 & 0 & 0 \\
\hline Vomiting & 3 & 1 & 0 & 0 & 0 \\
\hline Diarrhea & 3 & 0 & 1 & 0 & 4 \\
\hline Infection & 0 & 7 & 9 & 1 & 36 \\
\hline Esophagitis & 5 & 13 & 1 & 0 & 4 \\
\hline Radiation pneumonitis & 11 & 5 & 2 & 0 & 7 \\
\hline Elevation of serum creatinine & 0 & 0 & 0 & 0 & 0 \\
\hline Elevation of aminotransferases & 15 & 8 & 0 & 0 & 0 \\
\hline Abnormality of sodium balance & 21 & 0 & 4 & 0 & 14 \\
\hline Abnormality of potassium balance & 13 & 1 & 0 & 0 & 0 \\
\hline
\end{tabular}

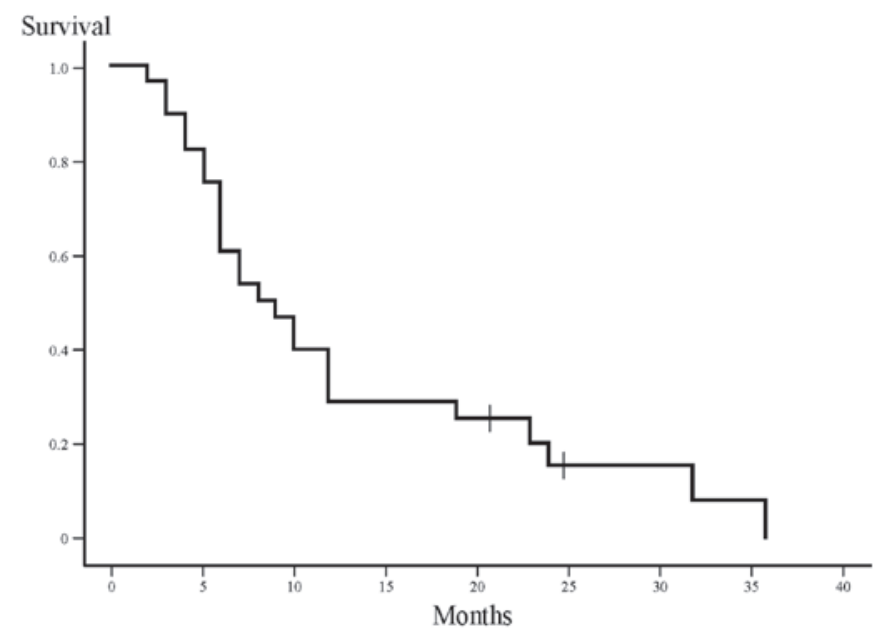

Figure 2.Progression-free survival (PFS) time estimated with the Kaplan-Meier method. The median PFS time was 8 months (range, 2-36 months).

best supportive care. Salvage chemotherapy was performed as follows: 7 patients received cytotoxic chemotherapy alone, 9 received both cytotoxic chemotherapy, and an EGFR tyrosine kinase inhibitor and 2 received an EGFR tyrosine kinase inhibitor alone.

Toxicity. The most commonly occurring toxicity was myelosuppression (Table II). Grade 3-4 hematological toxicities were frequently encountered: neutropenia, thrombocytopenia and anemia occurred in 100, 14 and $46 \%$ of the patients. Treatment with G-CSF was required during $48 \%$ of the courses (49 out of the 102 courses; median duration of administration, 3 days; range, 1-7 days). Three patients received erythrocyte transfusions. No patients received platelet transfusion.

The majority of the non-hematological toxicities were mild to moderate and transient. A total of 18 patients (64\%) presented with grade 1 or 2 radiation esophagitis and 1 patient (4\%) developed grade 3 radiation esophagitis, which resolved completely without residual dilation. No patients developed grade 4 radiation esophagitis. Grade 2 and 3 radiation pneumonitis requiring treatment with corticosteroids developed in 18 and $7 \%$ of the patients (5 and 2 out of the 28 patients), respectively. Corticosteroid therapy led to resolution of symptoms and radiographic abnormalities of the patients. Grade 3 and 4 infection occurred in 32 and $4 \%$ of the patients ( 9 and 1 out of the 28 patients), respectively. Eight patients had neutropenic fever which resolved quickly with antibiotic therapy and 1 patient had herpes zoster infection and recovered with antiviral therapy. One patient had bacterial pneumonia and required endotracheal intubation and ventilatory support. Although extubation was possible after the patient had received antibiotic treatment, steroids and oxygen, his condition gradually deteriorated and he expired 4 months after completion of chemoradiotherapy, without any recorded disease recurrence.

Dose intensity. The doses of carboplatin were not reduced. The doses of vinorelbine were reduced due to toxicity in 13 patients (46\%) (neutropenic fever in 9 and grade 4 neutropenia lasting $\geq 3$ days in 4 patients). During the 102 courses of chemotherapy, 6 $(6 \%)$ doses of vinorelbine were cancelled on day 8 , usually due to neutropenia. Out of the 102 courses of chemotherapy, 25 (25\%) were delayed, usually due to prolonged neutropenia. The actual delivered mean individual doses of vinorelbine and carboplatin were $16.8 \mathrm{mg} / \mathrm{m}^{2}$ (84\% of planned dose) and $2.35 \mathrm{mg} / \mathrm{ml} / \mathrm{min}$ (94\% of planned dose), respectively.

The patients were able to complete the radiotherapy according to the dose and schedule modification of the protocol. Radiotherapy was suspended (median, 4 days; range, 2-16 days) in $14(50 \%)$ patients due to grade 4 neutropenia (11 patients) and neutropenic fever or infection (3 patients).

\section{Discussion}

Platinum-based third-generation chemotherapeutic agents, such as vinorelbine, gemcitabine and paclitaxel should not 
be used at their full doses in concurrent chemoradiotherapy due to the high incidence of associated toxicity. Therefore, these agents have been used at reduced doses in previous clinical studies of concurrent chemoradiotherapy $(4,6,16)$. Furthermore, in previous phase III studies, platinum-based third-generation agents failed to demonstrate a survival benefit over platinum-based second-generation agents in concurrent chemoradiotherapy in patients with locally advanced NSCLC $(6,17)$. In addition, consolidation chemotherapy with docetaxel following concurrent chemoradiotherapy did not prolong survival; however, it increased the rates of toxicities, including pneumonitis (18). Moreover, gefitinib maintenance therapy following concurrent chemoradiotherapy and docetaxel consolidation showed lower survival rates compared to lack thereof (19). Furthermore, concurrent chemoradiotherapy in combination with bevacizumab was associated with a high incidence of tracheoesophageal fistulae formation (20). Thus far, a standard chemoradiotherapy regimen has not been determined, although concurrent chemoradiotherapy has been a standard treatment in patients with locally advanced NSCLC.

In previous phase III trials of concurrent chemoradiotherapy with new agents in patients with locally advanced NSCLC, the response rate range was $56-84 \%$, the median PFS range was 8-13.4 months and the MST range was 16.5-26.8 months $(6,16,17,21)$. In the present study, the overall response rate was $85.7 \%$, the median PFS time was 8 months and the MST was 23 months. Therefore, our data compare favorably with those of previously published trials in patients with locally advanced NSCLC.

The principal disadvantage of concurrent chemoradiotherapy is increased normal-tissue toxicity, particularly hematological, esophageal and pulmonary. In the present study, the most frequent toxicity was myelosuppression, particularly neutropenia. Grade 3-4 hematological toxicities included neutropenia, thrombocytopenia and anemia in 100, 14 and $46 \%$ of the patients, respectively. This high rate of myelosuppression may be associated with the use of carboplatin, which is strongly myelosuppressive, rather than cisplatin. However, the episodes of myelosuppression were manageable. Additionally, these toxicity rates compare favorably to those reported by previously published trials on patients with locally advanced NSCLC, in which the rates of grade 3-4 neutropenia, thrombocytopenia and anemia were $23-99 \%, 2-53 \%$ and $3-16 \%$, respectively $(6,16,17,21)$.

Severe esophagitis has been reported in many trials. The rate of grade 3-4 esophagitis in recent trials has ranged from $3-18 \%(6,16,17,21)$. In the present study, only 1 patient (4\%) developed grade 3 radiation esophagitis and no patients developed grade 4 esophagitis. In addition, severe radiation pneumonitis has been reported in several trials. The rates of grade 3-4 radiation pneumonitis in previous trials have been $1-10 \%(6,16,17,21)$. In our study, although grade 3 radiation pneumonitis developed in $7 \%$ of patients, corticosteroid therapy led to satisfactory resolution of symptoms and radiographic abnormalities of the patients. In the future, with the routine employment of modern radiotherapy technologies, such as 4-dimensional CT and respiration-gated radiotherapy, the rates of radiation-induced esophagitis and pneumonitis are expected to decrease, as improved imaging and radiotherapy delivery techniques may enable significant reductions in toxicity (22).
An important limitation in our study was the compromised accuracy of mediastinal lymph node staging, since mediastinoscopy and FDG PET were not mandatory in the staging work-up. In the present study, metastatic lymph nodes were defined as mediastinal lymph nodes $>10 \mathrm{~mm}$ along the short axis on CT. However, for the diagnosis of metastatic mediastinal lymph nodes, CT (sensitivity, 50-71\%; specificity, 66-89\%) is inferior to FDG PET (sensitivity, 67-91\%; specificity, 82-96\%) and mediastinoscopy (sensitivity, 80\%; specificity, 100\%) (23-25).

In conclusion, concurrent chemoradiotherapy with a divided schedule of carboplatin and vinorelbine is well-tolerated and effective in patients with locally advanced NSCLC. Therefore, this treatment is an acceptable option for patients with locally advanced NSCLC, particularly for patients who are not eligible for cisplatin-based chemoradiotherapy. Investigations are required for the design of more active regimens, including molecular-targeted therapies and modern radiotherapy technologies.

\section{References}

1. Goldstraw P, Crowley J, Chansky K, et al: International Association for the Study of Lung Cancer International Staging Committee and Participating Institutions. The IASCL Lung Cancer Staging Project: proposals for the revision of the TNM stage groupings in the forthcoming (seventh) edition of the TNM classification of malignant tumours. J Thorac Oncol 2: 706-714, 2007.

2. Auperin A, Le Pechoux C, Pignon JP, et al: Concomitant radio-chemotherapy based on platin compounds in patients with locally advanced non-small cell lung cancer (NSCLC): a metaanalysis of individual data from 1764 patients. Ann Oncol 17: 473-483, 2006.

3. Auperin A, Le Pechoux C, Rolland E, et al: Meta-analysis of concomitant versus sequential radiochemotherapy in locally advanced non-small-cell lung cancer. J Clin Oncol 28: 2181-2190, 2010.

4. Vokes EE, Herndon JE II, Crawford J, et al: Randomized phase II study of cisplatin with gemcitabine or paclitaxel or vinorelbine as induction chemotherapy followed by concomitant chemoradiotherapy for stage IIIB non-small-cell lung cancer: cancer and leukemia group B study 9431. J Clin Oncol 20: 4191-4198, 2002.

5. Go RS and Adjei AA: Review of the comparative pharmacology and clinical activity of cisplatin and carboplatin. J Clin Oncol 17: 409-422, 1999

6. Yamamoto N, Nakagawa K, Nishimura Y, et al: Phase III study comparing second-and third-generation regimens with concurrent thoracic radiotherapy in patients with unresectable stage III non-small-cell lung cancer: West Japan Thoracic Oncology Group WJTOG0105. J Clin Oncol 28: 3739-3745, 2010.

7. Hotta K, Matsuo K, Ueoka H, Kiura K, Tabata M and Tanimoto M: Meta-analysis of randomized clinical trials comparing cisplatin to carboplatin in patients with advanced non-small-cell lung cancer. J Clin Oncol 22: 3852-3859, 2004.

8. Ardizzoni A, Boni L, Tiseo M, et al: Cisplatin-versus carboplatin-based chemotherapy in first-line treatment of advanced non-small-cell lung cancer: an individual patient data metaanalysis. J Natl Cancer Inst 99: 847-857, 2007.

9. Douple EB, Richmond RC, O'Hara JA and Coughlin CT: Carboplatin as a potentiator of radiation therapy. Cancer Treat Rev 12: 111-124, 1985.

10. Edelstein MP, Wolfe LA III and Duch DS: Potentiation of radiation therapy by vinorelbine (Navelbine) in non-small cell lung cancer. Semin Oncol 23: 41-47, 1996.

11. Riedel RF, Andrews C, Garst J, et al: A phase II trial of carboplatin/vinorelbine with pegfilgrastim support for the treatment of patients with advanced non-small cell lung cancer. J Thorac Oncol 2: 520-525, 2007.

12. Bretti S, Manzin E, Celano A, Ritorto G, Loddo C and Berruti A: Low-dose carboplatin (AUC4.5) combined with vinorelbine in the treatment of advanced non-small cell lung cancer: A single institution phase II study. Oncol Rep 8: 381-385, 2001. 
13. Hoffman PC, Cohen EE, Masters GA, et al: Carboplatin plus vinorelbine with concomitant radiation therapy in advanced non-small cell lung cancer: a phase I study. Lung Cancer 38: 65-71, 2002.

14. Edelman MJ, Suntharalingam M, Burrows W, et al: Phase I/II trial of hyperfractionated radiation and chemotherapy followed by surgery in stage III lung cancer. Ann Thorac Surg 86: 903-911, 2008.

15. Masters GA, Hahn EA, Shevrin DH and Kies MS: Phase I/II trial of vinorelbine and divided-dose carboplatin in advanced non-small cell lung cancer. Lung Cancer 39: 221-226, 2003.

16. Zatloukal P, Petruzelka L, Zemanova M, et al: Concurrent versus sequential chemoradiotherapy with cisplatin and vinorelbine in locally advanced non-small cell lung cancer: a randomized study. Lung Cancer 46: 87-98, 2004.

17. Segawa Y, Kiura K, Takigawa N, et al: Phase III trial comparing docetaxel and cisplatin combination chemotherapy with mitomycin, vindesin, and cisplatin combination chemotherapy with concurrent thoracic radiotherapy in locally advanced non-small-cell lung cancer: OLCSG 0007. J Clin Oncol 28: 3299-3306, 2010.

18. Hanna N, Neubauer M, Yiannoutsos C, et al: Phase III study of cisplatin, etoposide, and concurrent chest radiation with or without consolidation docetaxel in patients with inoperable stage III non-small-cell lung cancer: the Hoosier Oncology Group and U.S. Oncology. J Clin Oncol 26: 5755-5760, 2008

19. Kelly K, Chansky K, Gaspar LE, et al: Phase III trial of maintenance gefitinib or placebo after concurrent chemoradiotherapy and docetaxel con $4=8$ solidation in inoperable stage III non-small-cell lung cancer: SWOG S0023. J Clin Oncol 26: 2450-2456, 2008.
20. Spigel DR, Hainsworth JD, Yardley DA, et al: Tracheoesophageal fistula formation in patients with lung cancer treated with chemoradiation and bevacizumab. J Clin Oncol 28: 43-48, 2009.

21. Furuse K, Fukuoka M, Kawahara M, et al: Phase III study of concurrent versus sequential thoracic radiotherapy in combination with mitomycin, vindesin, and cisplatin in unresectable stage III non-small-cell lung cancer. J Clin Oncol 17: 2692-2699, 1999.

22. Haasbeek CJ, Slotman BJ and Senan S: Radiotherapy for lung cancer: clinical impact of recent technical advances. Lung Cancer 64: 1-8, 2009.

23. Gould MK, Kuschner WG, Rydzak CE, et al: Test performance of positron emission tomography and computed tomography for mediastinal staging in patients with non-small-cell lung cancer. Ann Intern Med 139: 879-892, 2003.

24. Birim O, Kappetein AP, Stijnen T and Bogers AJ: Metaanalysis of positron emission tomographic and computed tomographic imaging in detecting mediastinal lymph node metastases in non-small cell lung cancer. Ann Thorac Surg 79: 375-381, 2005.

25. Detterbeck FC, Jantz MA, Wallace M, Vansteenkiste J and Silvestri GA: American College of Chest Physicians. Invasive mediastinal staging of lung cancer: AACP evidence-based clinical practice guidelines (2nd Edition). Chest 132: S202-S220, 2007. 